\title{
Possible IgG4-Related Kidney Disease Requiring a Differential Diagnosis of Membranous Lupus Nephritis
}

\author{
Mayumi Yahata ${ }^{1}$, Satoko Takahashi ${ }^{1}$, Izaya Nakaya ${ }^{1}$, Tsutomu Sakuma ${ }^{2}$, \\ Hiroshi Sato ${ }^{3}$ and Jun Soma ${ }^{1}$
}

\begin{abstract}
A 25-year-old woman presented with fever, arthralgia and proteinuria exhibiting leukopenia, hypocomplementemia, increased serum IgG and IgG4, and positive antinuclear and anti-double-stranded DNA antibodies. Renal biopsy revealed membranous nephropathy with tubulointerstitial nephritis. IgG subclass immunofluorescence revealed intense IgG4 expression in glomeruli, but no expression of IgG2. Observations resembled membranous lupus nephritis with tubulointerstitial nephritis; however, elevated IgG4, low titers of antinuclear and anti-double-stranded DNA antibodies, IgG4-bearing cell infiltration, and characteristic IgG subclass deposition in glomeruli prompted diagnosis of IgG4-related tubulointerstitial nephritis with membranous nephropathy. It is challenging but important to distinguish lupus nephritis from IgG4-related kidney disease.
\end{abstract}

Key words: IgG4-related kidney disease, lupus nephritis, IgG4-related tubulointerstitial nephritis, membranous nephropathy

(Intern Med 51: 1731-1736, 2012)

(DOI: 10.2169/internalmedicine.51.7549)

\section{Introduction}

The renal pathology of IgG4-related disease comprises tubulointerstitial nephritis with IgG4-positive plasma cell and lymphocyte infiltration into the renal interstitium and fibrosis (1). Hypocomplementemia and elevated antinuclear antibody (ANA) are frequently observed, whereas anti-SS-A, anti-SS-B, anti-Sm, anti-RNP and anti-double-stranded DNA (dsDNA) antibodies are generally negative (1). Occasionally, IgG 4-related tubulointerstitial nephritis accompanies glomerular diseases such as membranous nephropathy, mesangial proliferative glomerulonephritis and membranoproliferative glomerulonephritis; however, membranous nephropathy is dominant (2-12). We report a case, initially considered to be IgG4-related tubulointerstitial nephritis with membranous nephropathy requiring a differential diagnosis of membranous lupus nephritis (Class V) with severe tubulointerstitial changes.

\section{Case Report}

A 25-year-old woman, with no significant medical history, visited our hospital with fever, malaise and arthralgia lasting 1 month and erythema on her right palm for 6 months. We suspected collagen abnormalities, on the basis of leukopenia, proteinuria, and low presence of ANA. Referred to our renal division, she was admitted for further examination in February 2010.

On admission, the patient's blood pressure was 107/78 $\mathrm{mmHg}$, body temperature was $37.1^{\circ} \mathrm{C}$, height was $156.9 \mathrm{~cm}$, and weight was $43.8 \mathrm{~kg}$ (a 5-kg loss over 3 months). Physical examination revealed erythema on her right palm and bilateral lower extremity edema. Pulmonary rales were heard bilaterally. Urinalysis revealed proteinuria of $2.7 \mathrm{~g} / \mathrm{day}$. Urinary sediment examination showed 1-4 red blood cells/high power field and urinary $\beta 2$-microglobulin was $234 \mu \mathrm{g} / \mathrm{L}$ (normal, $<230 \mu \mathrm{g} / \mathrm{L}$ ). Hematologic findings were as follows: erythrocyte count, $397 \times 10^{4} / \mu \mathrm{L}$; hemoglobin, $10.8 \mathrm{~g} / \mathrm{dL}$; leu-

\footnotetext{
${ }^{1}$ Division of Nephrology, Iwate Prefectural Central Hospital, Japan, ${ }^{2}$ Division of Pathology, Iwate Prefectural Central Hospital, Japan and ${ }^{3}$ Department of Clinical Pharmacology and Therapeutics, Tohoku University Graduate School of Pharmaceutical Sciences and Medicine, Tohoku University, Japan

Received for publication February 17, 2012; Accepted for publication March 27, 2012

Correspondence to Dr. Mayumi Yahata, myahata@chuo-hp.jp
} 


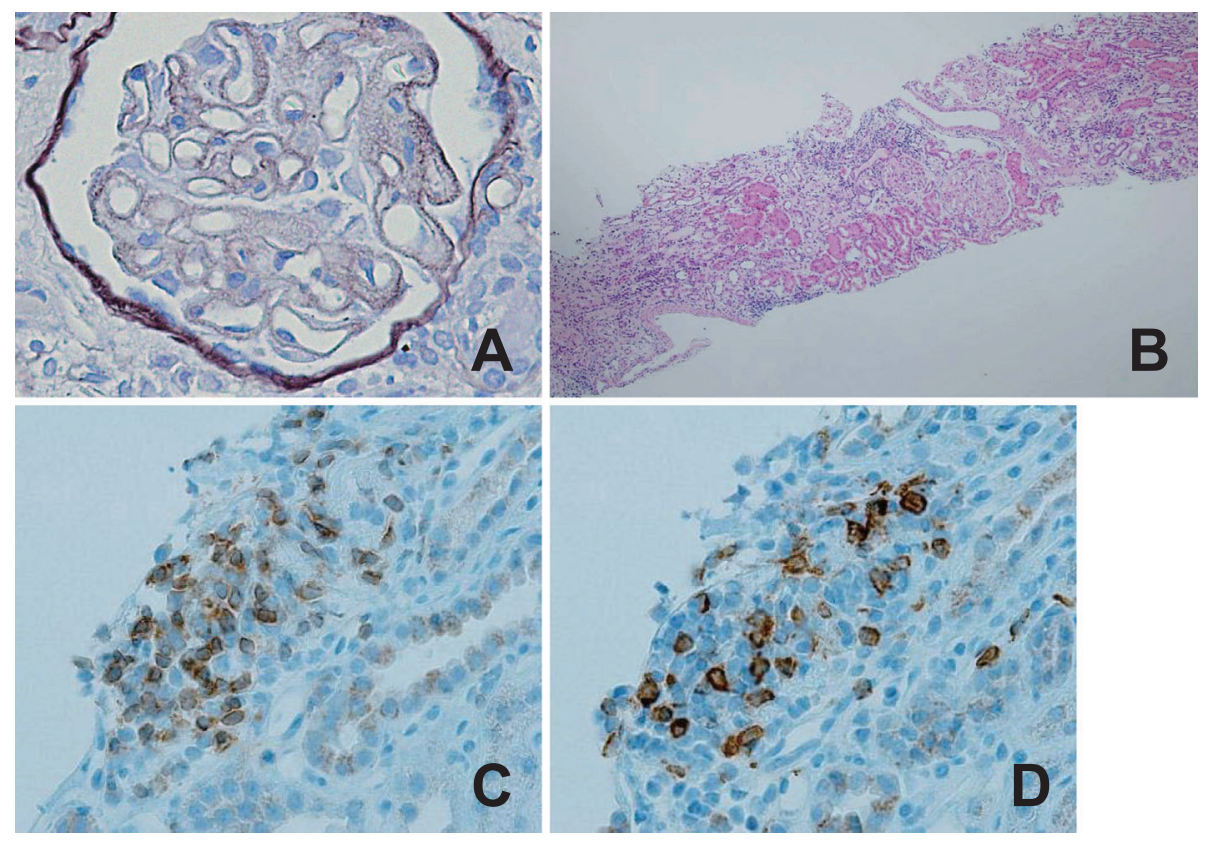

Figure 1. Light microscopy shows (A) diffuse thickened glomerular capillary walls with small spikes and a worm-eaten appearance, mild increase in mesangial matrix, and mononuclear cell infiltration around the glomerulus. (B) Tubular atrophy and diminishment are observed associated with mononuclear cell infiltration in $\mathbf{5 0 \%}$ of the interstitium. The distribution of mononuclear cell infiltration is patchy. Immunoperoxidase stainings of (C) IgG and (D) IgG4 show that most of IgG-positive infiltrating cells are IgG4 positive. (A) Periodic acid-Schiff stain, and (B) Hematoxylin and Eosin staining. Original magnification: $(A) \times 400 ;(B) \times 40$; and $(C)$ and $(D) \times 400$.

kocyte count, 2,690/ $\mathrm{LL}$; platelet count, $20.4 \times 10^{4} / \mu \mathrm{L}$. Blood chemistry tests showed the following: total protein, $7.5 \mathrm{~g} /$ $\mathrm{dL}$; albumin, $3.7 \mathrm{~g} / \mathrm{dL}$; blood urea nitrogen, $14.8 \mathrm{mg} / \mathrm{dL}$; creatinine, $0.64 \mathrm{mg} / \mathrm{dL}$; aspartate aminotransferase, $31 \mathrm{IU} / \mathrm{L}$; alanine aminotransferase, 29 IU/L. Serological studies revealed the following: serum $\mathrm{IgG}, 2,253 \mathrm{mg} / \mathrm{dL}$ (normal, 870-1,700 mg/dL); serum IgA, $292 \mathrm{mg} / \mathrm{dL}$; serum IgM, 134 $\mathrm{mg} / \mathrm{dL}$; serum IgG1, 1,050 mg/dL (normal, 320-748 mg/dL); IgG2, $803 \mathrm{mg} / \mathrm{dL}$ (normal, 208-754 mg/dL); IgG3, $83 \mathrm{mg} /$ $\mathrm{dL}$ (normal, 6.6-88.3 mg/dL); IgG4, $317 \mathrm{mg} / \mathrm{dL}$ (normal, 4.8-105 mg/dL); Complement component (C) $3,56 \mathrm{mg} / \mathrm{dL}$ (normal, 63-134 mg/dL); C4, $23 \mathrm{mg} / \mathrm{dL}$ (normal, 13-36 mg/ $\mathrm{dL}$ ); hemolytic complement activity, $37.1 \mathrm{U} / \mathrm{mL}$ (normal, 30-50 U/mL); ANA, $\times 40$ (speckled); anti-dsDNA antibody, $25 \mathrm{IU} / \mathrm{mL}$ (normal, <12 IU/mL). Anti-SS-A and B antibodies, anti-Sm antibody, myeloperoxidase- and proteinase 3antineutrophil cytoplasmic antibodies were negative. Computed tomography from chest to pelvis showed normal-sized kidneys and no abnormalities of the pancreas, salivary glands, or retroperitoneum, but revealed bilateral interstitial reticular markings in the chest and swollen axillary and cervical lymph nodes.

Renal biopsy was performed on the third day of admission. The specimen exhibited 17 glomeruli on light microscopy, three of which were globally sclerotic, whereas others showed alterations including mild increase in mesangial matrix, globally-thickened capillary walls with spikes, wormeaten appearance and partial double contours (Fig. 1A). In- terstitial fibrosis with infiltration of lymphocytes and plasma cells was observed in $>50 \%$ of the interstitium, and atrophic and hypertrophic tubules were mixed (Fig. 1B). Routine immunofluorescence (IF) using frozen sections (Fig. 2) revealed marked intensity $(+++)$ for $\mathrm{IgG}$, moderate intensity $(++)$ for $\operatorname{IgM}$ and $\mathrm{C} 3$, and mild intensity (+) for $\operatorname{IgA}$ and $\mathrm{C} 1 \mathrm{q}$, which were found diffusely and globally along the capillary walls and tubular basement membranes in a granular pattern. These deposits were partially evident in the mesangium. Additionally, we performed immunoperoxidase staining for IgG and IgG4 using paraffin-embedded sections stained with mouse anti-human monoclonal IgG (Nichirei Bioscience Inc., Tokyo, Japan) and mouse anti-human monoclonal IgG4 (The Binding Site Group Ltd., Birmingham, UK) using an immunostaining system Ventana HX Benchmark (Roche Diagnostics Japan, Tokyo, Japan). Frozen sections were further stained with mouse monoclonal antibodies specific to IgG subclasses 1-4 (The Binding Site Group Ltd.), followed by polyclonal fluorescein isothiocyanate (FITC)-conjugated rabbit anti-mouse antibody (Dako Japan, Kyoto, Japan). All monoclonal antibodies for IgG subclasses were used at a dilution of 1:100. Immunoperoxidase staining showed that almost of IgG-positive plasma cells were IgG4positive cells (Fig. 1C, D). IF for IgG subclasses showed strong and diffuse IgG4 expression along the capillary walls and tubular basement membranes, and partial mesangial expression. IgG1 and IgG3 were weak relative to IgG4, whereas IgG2 was absent (Fig. 2). Subepithelial electron- 

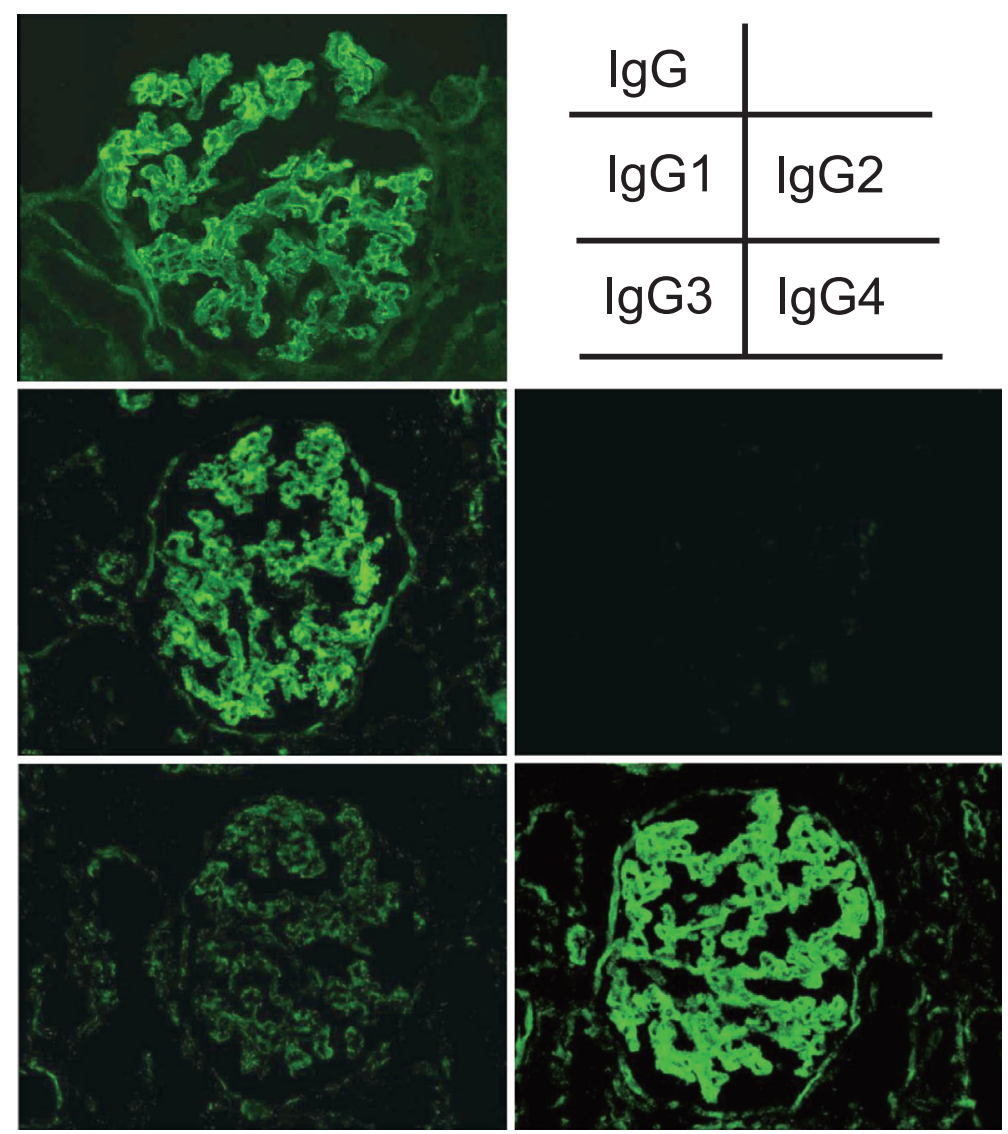

Figure 2. Immunofluorescence of IgG and IgG subclasses. Marked, diffuse granular deposits of IgG are evident along the glomerular capillary walls and in part of the mesangium. Among IgG subclasses, IgG4 is dominant, followed by IgG1 and IgG3. IgG2 is absent. Original magnification: ×250.

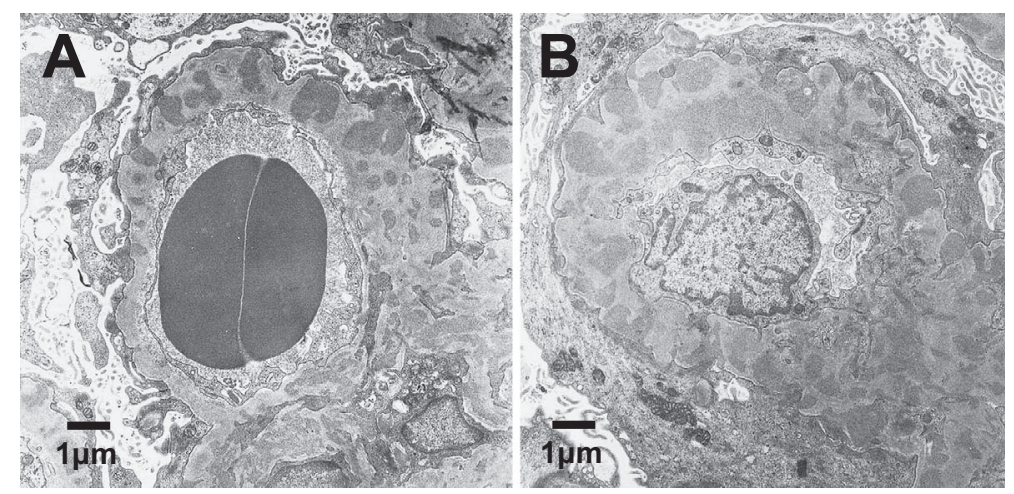

Figure 3. Electron microscopy shows (A) subepithelial electron-dense deposits of various sizes with diffuse distribution. In addition to subepithelial deposits, (B) subendothelial and mesangial electron-dense deposits are evident with segmental distribution.

dense deposits were diffusely observed on electron microscopy (Fig. 3A). Mesangial and subendotherial electron-dense deposits were also observed with segmental distribution (Fig. 3B).

Although a definitive diagnosis of either lupus membranous nephritis with tubulointerstitial nephritis or IgG4related tubulointerstitial nephritis with membranous nephropathy was not reached, $20 \mathrm{mg} /$ day oral prednisolone was commenced under a provisional diagnosis of IgG4-related kidney disease. Proteinuria resolved after 2 weeks, and pred- nisolone was tapered to $2.5 \mathrm{mg} /$ day. Proteinuria, ANA and anti-dsDNA antibody remained negative, and leukocyte count fluctuated between 3,000 and $4,000 / \mu \mathrm{L}$.

\begin{tabular}{l} 
Discussion \\
\hline The renal pathology of IgG4-related disease usually com- \\
prises tubulointerstitial nephritis associated with IgG4- \\
positive plasma cells and lymphocyte infiltration into the re- \\
nal interstitium with fibrosis (1). In some cases, membra-
\end{tabular}


nous nephropathy, mesangial proliferative glomerulonephritis, and membranoproliferative glomerulonephritis associated with IgG4-related tubulointerstitial nephritis have been reported (2-12). Hypocomplementemia and ANA presence are frequently observed (1). This case met the diagnostic criteria for IgG4-related kidney disease of the Japanese Society of Nephrology (13) as follows:

- presence of some kidney damage with elevated serum IgG level and hypocomplementemia;

- elevated serum IgG4 level (IgG4 $\geq 135 \mathrm{mg} / \mathrm{dL})$; and

- histological findings of dense lymphoplasmacytic infiltration with infiltrating IgG4-positive plasma cells $>10$ / high power field and/or IgG4/IgG-positive plasma cells $>40 \%$, and characteristic fibrosis surrounding nests of lymphocytes and/or plasma cells.

The present case lacked other organ involvement such as autoimmune pancreatitis, sialadenitis, and sclerocholangitis, which are frequently observed in IgG4-related kidney disease $(1,2)$. In addition, the distribution of interstitial lesions seemed to be patchy rather than showing the characteristic regional lesion distribution $(1,2)$. However, these do not always rule out IgG4-related kidney disease, because 1) this case showed bilateral interstitial reticular markings in the lung and swollen axillary and cervical lymph nodes, which are considered to be findings associated with IgG4-related systemic disease $(2) ; 2$ ) a few cases with renal lesion alone were also reported $(8,14)$; and 3$)$ it is possible that this case was at an early stage of the disease when characteristic findings of a marked and unique fibrosis was not observed (2).

Taken together, it was highly possible that this case was equivalent to IgG4-related kidney disease. However, we diagnosed this case as "possible" IgG4-related tubulointerstitial nephritis concomitant with membranous nephropathy. Our diagnosis was a "possible" but not "definite" case because of difficulty differentiating our findings from membranous lupus nephritis with severe tubulointerstitial changes; the case also fulfills the criteria for systemic lupus erythematosus by the 1997 update of the 1982 American College of Rheumatology Revised Criteria for Classification of Systemic Lupus Erythematosus as follows:

- leukopenia;

- renal disorder;

- presence of anti-dsDNA antibody; and

- presence of ANA.

All glomeruli showed globally thickened capillary walls with spikes, a worm-eaten appearance, and partial double contours associated with mild mesangial proliferation. Immunofluorescence revealed full house patterns in the glomeruli, whereas electron microscopy diffusely showed subepithelial electron-dense deposits with partial subendothelial and mesangial deposits. These histopathological findings are not inconsistent with those of lupus nephritis Class $\mathrm{V}$, on the basis of the International Society of Nephrology/ Renal Pathology Society classification (15).

Severe tubulointerstitial nephritis in systemic lupus erythematosus is most prevalent in patients with Class IV lupus nephritis (16). However, in systemic lupus erythematosus, some cases show severe tubulointerstitial nephritis with minimal glomerular changes or mild mesangial proliferation (17-27), although rare. To date, 12 cases have been reported. Among them, Omokawa et al. (27) reported a case of Class II lupus nephritis with predominant tubulointerstitial nephritis, in which IgG subclass analysis showed glomerular IgG1, IgG2, and IgG4 deposition, positive staining for IgG4 in peritubular interstitium and along the tubular basement membranes, and abundant IgG1-, IgG3-, and IgG4-positive plasma cells in the interstitium. In contrast, in the present case, IgG4 was the strongest of the four subclasses, whereas IgG2 deposition was absent. This pattern entirely differs from that typical in membranous lupus nephritis, in which IgG2 deposition is more frequent than $\mathrm{IgG} 3$ and $\mathrm{IgG} 4$, and intensity of $\mathrm{IgG} 4$ deposition is lower than that of $\operatorname{IgG} 1$ and $\operatorname{IgG} 2(28,29)$. Partial mesangial deposition was also negative for $\mathrm{IgG} 2$ in the present case; conversely, in the case of Class II lupus nephritis with severe tubulointerstitial nephritis reported by Omokawa et al. (27), mesangial IgG2 deposition was pronounced.

Glomerular changes were evident in a quarter of the reported cases of IgG4-related tubulointerstitial nephritis (2). Among them, membranous nephropathy was dominant (2-12). Membranous nephropathy, concomitant with IgG4-related tubulointerstitial nephritis, is considered linked to IgG4-related disease, but not a primary glomerular disease, because circulating anti-phospholipase $\mathrm{A}_{2}$ receptor antibodies, the most common autoantibodies in idiopathic membranous nephropathy (30), were undetected $(11,12)$. One hypothesis of membranous nephropathy development in IgG4-ralated disease is that proliferating plasma cells produce IgG4 that is autoreactive against podocytes antigens (12). In addition to the absence of anti-phospholipase $\mathrm{A}_{2}$ receptor antibodies, Cravedi et al. (11), in a case of membranous nephropathy concomitant with IgG4-related disease, reported that $\mathrm{IgG} 4$, but not $\mathrm{IgG} 2$ was evident on glomerular capillary walls. Saeki et al. (8) also reported a similar pattern of IgG subclass deposition in IgG4-related membranous nephropathy. These are very interesting because the IgG subclass deposition pattern in these cases closely resembles our findings.

Both of ANA and anti-dsDNA antibody were present in the current case. However, their titers were very low, at $\times 40$ and $25 \mathrm{IU} / \mathrm{mL}$ (normal, $<12 \mathrm{IU} / \mathrm{mL}$ ), respectively. Low titer of anti-dsDNA antibody is reportedly less specific for lupus, and other diseases, such as hepatitis, rheumatoid arthritis, drug-induced disorders, infection and myeloma, should be considered (31-33). Raissian et al. (34) summarized clinicopathological studies of 35 patients with IgG4-related tubulointerstitial nephritis. Among them, two tested positive for anti-dsDNA antibody.

Together, these histopathological and laboratory findings suggest that membranous lupus nephritis with severe tubulointerstitial nephritis was less likely in this case. To our 
knowledge, there are no reported cases of severe tubulointerstitial nephritis associated with membranous lupus nephritis. Additionally, a good response to moderate-dose steroid therapy supports a diagnosis of IgG4-related kidney disease (2).

In summary, it is challenging but important to distinguish IgG4-related kidney disease from lupus nephritis because of their clinical and histopathological similarities. Previous cases diagnosed as lupus nephritis, before recognition of IgG4-related systemic disease (35), could include a considerable number of cases of IgG4-related kidney disease. We thought it is highly possible that this case was IgG4-related tubulointerstitial nephritis concomitant with membranous nephropathy, but not membranous lupus nephritis, because titers of ANA and anti-dsDNA antibody were very low, a moderate dose of prednisolone was very effective, and IgG subclass deposition pattern differed from that of lupus nephritis. However, few studies describe IgG subclass deposition pattern in glomeruli of IgG4-related kidney disease. Further studies are necessary to clearly distinguish IgG4related kidney disease from lupus nephritis.

\section{The authors state that they have no Conflict of Interest (COI).}

\section{Acknowledgement}

The authors thank Kiyomi Kisu and Shuichi Futatsumori for their excellent technical assistance. This work was presented at the 41st Eastern Regional Meeting of the Japanese Society of Nephrology, September 14-15, 2011, Tokyo, Japan.

\section{References}

1. Saeki T, Nishi S, Imai N, et al. Clinicopathological characteristics of patients with IgG4-related tubulointerstitial nephritis. Kidney Int 78: 1016-1023, 2010.

2. Nishi S, Imai N, Yoshida K, Ito Y, Saeki T. Clinicopathological findings of immunoglobulin G4-related kidney disease. Clin Exp Nephrol 15: 810-819, 2011.

3. Takeda S, Haratake J, Kasai T, Takaeda C, Takazakura E. IgG4associated idiopathic tubulointerstitial nephritis complicating autoimmune pancreatitis. Nephrol Dial Transplant 19: 474-476, 2004.

4. Uchiyama-Tanaka Y, Mori Y, Kimura T, et al. Acute tubulointerstitial nephritis associated with autoimmune-related pancreatitis. Am J Kidney Dis 43: e18-e25, 2004.

5. Watson SJ, Jenkins DA, Bellamy CO. Nephropathy in IgG4 related systemic disease. Am J Surg Pathol 30: 1472-1477, 2006.

6. Cornell LD, Chicano SL, Deshpande V, et al. Pseudotumors due to IgG4 immune-complex tubulointerstitial nephritis associated with autoimmune pancreatocentric disease. Am J Surg Pathol 31: 1586-1597, 2007

7. Morimoto J, Hasegawa Y, Fukushima H, et al. Membranoproliferative glomerulonephritis-like glomerular disease and concurrent tubulointerstitial nephritis complicating IgG4-related autoimmune pancreatitis. Intern Med 48: 157-162, 2009.

8. Saeki T, Imai N, Ito T, Yamazaki H, Nishi S. Membranous nephropathy associated with IgG4-related systemic disease and without autoimmune pancreatitis. Clin Nephrol 71: 173-178, 2009.

9. Saida Y, Homma N, Hama H, et al. Case of IgG4-related tubulointerstitial nephritis showing the progression of renal dysfunction after a cure for autoimmune pancreatitis. Nihon Jinzo Gakkai Shi 52: 73-79, 2010 (in Japanese).

10. Katano K, Hayatsu Y, Matsuda T, et al. Endocapillary proliferative glomerulonephritis with crescent formation and concurrent tubulointerstitial nephritis complicating retroperitoneal fibrosis with a high serum level of IgG4. Clin Nephrol 68: 308-314, 2007.

11. Cravedi P, Abbate M, Gagliardini E, et al. Membranous nephropathy associated with IgG4-related disease. Am J Kidney Dis 58: 272-275, 2011.

12. Fervenza FC, Downer G, Beck LH Jr, Sethi S. IgG4-related tubulointerstitial nephritis with membranous nephropathy. Am J Kidney Dis 58: 320-324, 2011.

13. Kawano M, Saeki T, Nakashima H, et al. Proposal for diagnostic criteria for IgG4-related kidney disease. Clin Exp Nephrol 15: 615-626, 2011.

14. Tsubata Y, Akiyama F, Oya T, et al. IgG4-related chronic tubulointerstitial nephritis without autoimmune pancreatitis and the time course of renal function. Intern Med 49: 1593-1598, 2010.

15. Weening JJ, D'Agati VD, Schwartz MM, et al. The classification of glomerulonephritis in systemic lupus erythematosus revisited. J Am Soc Nephrol 15: 241-250, 2004.

16. Yu F, Wu LH, Tan Y, et al. Tubulointerstitial lesions of patients with lupus nephritis classified by the 2003 International Society of Nephrology and Renal Pathology Society system. Kidney Int 77: 820-829, 2010

17. Epstein FH, Wittenberg J, Coggins $\mathrm{CH}$, McCluskey RT, Lichtenstein NS. Case records of the Massachusetts General Hospital. Weekly clinicopathological exercise. Case 2 1976. N Engl J Med 294: 100-105, 1976.

18. Disler PB, Lewin JR, Laidley L, Meyers AM. Systemic lupus erythematosus with pure interstitial disease: a case report (abstract). Kidney Int 13: 428, 1978.

19. Cunningham E, Provost $T$, Brentjens J, Reichlin M, Venuto RC. Acute renal failure secondary to interstitial lupus nephritis. Arch Intern Med 138: 1560-1561, 1978.

20. Tron F, Ganeval D, Droz D. Immunologically-mediated acute renal failure of nonglomerular origin in the course of systemic lupus erythematosus [SLE]. Report of two cases. Am J Med 67: 529$532,1979$.

21. Klahr S, Lynch R. Clinicopathological conference: interstitial nephritis in a patient with systemic lupus erythematosus. Am J Med 69: 775-781, 1980.

22. Makker SP. Tubular basement membrane antibody-induced interstitial nephritis in systemic lupus erythematosus. Am J Med 69: 949-952, 1980.

23. Gur H, Kopolovic Y, Gross DJ. Chronic predominant interstitial nephritis in a patient with systemic lupus erythematosus: a follow up of three years and review of the literature. Ann Rheum Dis 46: 617-623, 1987

24. Singh AK, Ucci A, Madias NE. Predominant tubulointerstitial lupus nephritis. Am J Kidney Dis 27: 273-278, 1996.

25. Michail S, Stathakis Ch, Marinaki S, Revenas C, Nakapoulou L, Vaiopoulos G. Relapse of predominant tubulointerstitial lupus nephritis. Lupus 12: 728-729, 2003.

26. Mori Y, Kishimoto N, Yamahara H, et al. Predominant tubulointerstitial nephritis in a patient with systemic lupus nephritis. Clin Exp Nephrol 9: 79-84, 2005.

27. Omokawa A, Wakui H, Okuyama S, et al. Predominant tubulointerstitial nephritis in a patient with systemic lupus erythematosus: phenotype of infiltrating cells. Clin Nephrol 69: 436-444, 2008.

28. Imai $\mathrm{H}$, Hamai $\mathrm{K}$, Komatsuda $\mathrm{A}$, Ohtani H, Miura AB. IgG subclasses in patients with membranoproliferative glomerulonephritis, membranous nephropathy, and lupus nephritis. Kidney Int 51: 270-276, 1997.

29. Kuroki A, Shibata T, Honda H, Totsuka D, Kobayashi K, Sugisaki T. Glomerular and serum IgG subclasses in diffuse proliferative lupus nephritis, membranous lupus nephritis, and idiopathic membranous nephropathy. Intern Med 41: 936-942, 2002. 
30. Beck LH Jr, Bonegio RG, Lambeau G, et al. M-type phospholipase A2 receptor as target antigen in idiopathic membranous nephropathy. N Engl J Med 361: 11-21, 2009.

31. Isenberg DA, Manson JJ, Ehrenstein MR, Rahman A. Fifty years of anti-ds DNA antibodies: are we approaching journey's end? Rheumatology (Oxford) 46: 1052-1056, 2007.

32. Rekvig OP, Nossent JC. Anti-double-stranded DNA antibodies, nucleosomes, and systemic lupus erythematosus: a time for new paradigms? Arthritis Rheum 48: 300-312, 2003.
33. Nossent HC, Rekvig OP. Is closer linkage between systemic lupus erythematosus and anti-double-stranded DNA antibodies a desirable and attainable goal? Arthritis Res Ther 7: 85-87, 2005.

34. Raissian Y, Nasr SH, Larsen CP, et al. Diagnosis of IgG4-related tubulointerstitial nephritis. J Am Soc Nephrol 22: 1343-1352, 2011.

35. Hamano H, Kawa S, Horiuchi A, et al. High serum IgG4 concentrations in patients with sclerosing pancreatitis. $\mathrm{N}$ Engl $\mathrm{J}$ Med 344: 732-738, 2001.

(C) 2012 The Japanese Society of Internal Medicine http://www.naika.or.jp/imindex.html 